\title{
Wide Dynamic Range Detection and All That...
}

\author{
Sol M. Gruner
}

Physics Dept. \& CHESS, Cornell University, Ithaca, NY 14853-2501 smg26@cornell.edu

Imaging radiation detectors with enhanced capabilities, such as those with wider dynamic ranges, are being designed to take advantage of beams from $x$-ray free electron lasers, low emittance storage rings, and aberration-corrected electron microscopes. While users intuitively know they want a detector with a wide dynamic range, it is often unclear as to the dynamic range that may be required for a given type of experiment. The situation is further confused by the fact that various, nonequivalent definitions of dynamic range are in common use. The practical dynamic range of an imaging detector is indelibly intertwined with other detector characteristics, such as sensitivity, instantaneous count-rate, Poisson statistics, and calibrations. I will discuss the dynamic range in the context of $x$-ray and electron microscope detectors, and give example of experiments where a wide dynamic range is both required, and greatly extends scientific capabilities. 